\title{
Les hauts et les bas du financement canadien au HCR : quelle aide et pour quels réfugiés?
}

\author{
CATHERINE-LUNE GRAYSON ET FRANÇOIS AUDET
}

\section{Résumé}

Le Canada compte parmi les donateurs importants du Haut Commissariat des Nations Unies pour les réfugiés (HCR). Entre 2006 et 2013, le financement canadien au HCR a plus que doublé, pour atteindre 84,9 millions de dollars canadiens en 2013. Durant la même période, ce financement a de plus en plus été affecté à un pays donné. Cet article examine ce qui motive à la fois la quantité et la qualité de la contribution canadienne au HCR durant cette période. Ce faisant, les auteurs abordent la relation entre le financement du pays et sa politique étrangère et intérieure, ainsi que l'éventuelle influence canadienne sur le HCR.

\section{Abstract}

Canada has long been one of UNHCR's important donors. Between 2006 and 2013, the country's contribution to the agency has more than doubled, reaching CAN\$84.9 million in 2013. During the same period, Canada's funding has been increasingly earmarked for specific country operations. This article examines what motivates the quantity and quality of Canada's contributions to UNHCR. In doing so, it discusses the relationship between the country's funding and its foreign and national policy, as well as its ability to influence UNHCR.

\section{Introduction}

T e Canada compte généralement parmi les dix premiers donateurs du Haut Commissariat des Nations Unies pour les réfugiés (HCR). Entre 2006 et 2013, le financement du Canada au HCR a plus que doublé, passant de 37,3 à 84,9 millions de dollars canadiens (en dollars constants de $2013^{1}$ ), soit une augmentation de $128 \%$. Durant cette période, le financement canadien à l'ensemble de l'Organisation des
Nations Unies (ONU), incluant ses organes, ses institutions spécialisées, ses fonds et ses programmes, n’a crû que de $34 \%$. Cette croissance est comparable à celle de l'aide multilatérale (37\%) qui inclut le financement à l'oNu, mais également à d'autres organisations internationales comme des institutions financières internationales et régionales, l'Organisation internationale de la francophonie ou encore l'Organisation internationale pour les migrations. L'aide internationale totale (ou aide publique au développement) qui combine les aides bilatérale et multilatérale a augmenté entre 2006 et 2010, mais diminué depuis 2011 pour être ramenée en 2013 à un niveau comparable à celui de 2006. Par conséquent, alors que le financement multilatéral en général, et à l'oNu en particulier, est en croissance légère et que le financement bilatéral est en baisse, les fonds octroyés au HCR connaissent une augmentation significative ${ }^{2}$. Sur la même période, selon les données compilées par le HCR, la part de la contribution canadienne affectée à un pays, une région ou un secteur a augmenté de $32 \%$. Ainsi, la proportion des fonds canadiens dont peut disposer librement le HCR, des fonds communément décrits comme "non affectés»3, a considérablement diminué. Ces constats reflètent une tendance globale: le financement des donateurs importants, parmi lesquels le Canada, a augmenté durant la dernière décennie, tout comme le budget du HCR. Au même moment cependant, les pays donateurs affectent de plus en plus leur contribution à une zone géographique, à un thème ou à secteur d'activité spécifiques.

Ces observations soulèvent un certain nombre de questions quant aux raisons de ce financement certes en croissance, mais de nature de plus en plus restrictive. En examinant les tendances du financement canadien au HCR entre 2006 et 2013, cet article s'interroge sur la relation entre ce financement, la politique étrangère canadienne et la politique nationale d'immigration et d'asile, ainsi que sur la relation 
que le Canada entretient avec le HCR. Cette étude est de nature exploratoire et ne prétend, par conséquent, pas fournir des réponses définitives. Elle espère plutôt ouvrir des pistes de réflexion qui pourraient inspirer de futures recherches. Il convient de noter que durant la période analysée, le gouvernement conservateur de Stephen Harper était au pouvoir, soit un gouvernement qui a marqué une certaine rupture dans la politique étrangère canadienne, notamment en adoptant une position très critique envers les Nations Unies 4.

La littérature sur le financement des organisations internationales est étonnamment limitée, en particulier si l'on considère que cette question est déterminante dans leur fonctionnement et dans le débat sur leur autonomie 5 . Dans le cas du HCR, cette question est d'autant plus pertinente que la quasi-totalité de son budget ( $98 \%$ ) dépend de contributions volontaires, principalement étatiques. Les articles de Väyrynen en $2001^{6}$ et de Roper et Barria en $2010^{7}$ comptent parmi les rares études consacrées spécifiquement à ce sujet. Le premier se penche sur le budget du HCR et souligne que le financement octroyé à l'agence varie selon les intérêts politiques des donateurs. Väyrynen observe que dès les années 1990, la proportion du financement affecté par les États augmente, une tendance qu'il attribue à leur volonté de contrôler les interventions du HCR en fonction de leurs intérêts nationaux, mais également à leur manque de confiance envers l'institution. Roper et Barria étudient la nature du financement au HCR et soulignent que les États tirent des bénéfices privés de leurs contributions. Loescher, Betts et Milner ${ }^{8}$ abordent pour leur part la question du financement de manière connexe. Ils affirment que les motivations des États à financer le HCR sont complexes : ceux-ci souhaitent respecter leurs obligations internationales et leurs obligations morales, mais ils font également des choix intéressés. Les contributions d'États affectées à un pays donné s'expliquent plus souvent par une connexion historique avec le pays, leur politique étrangère, leurs diasporas, des préoccupations sécuritaires, migratoires ou commerciales que par une stricte volonté de protéger les populations déplacées. Dès lors, le financement au HCR, et plus largement le financement humanitaire ${ }^{9}$, se trouve à la jonction des politiques intérieures et extérieures, soit des politiques intrinsèquement liées. Depuis la fin de la guerre froide, Smillie et Minear ${ }^{10}$ observent que la politique intérieure oriente toujours davantage le comportement des États donateurs, États qui ont généralement sanctionné des politiques d'asile et migratoires de plus en plus strictes et dissuasives pour se protéger des populations réfugiées présentées comme menaçantes, hors norme ${ }^{11}$. Laide humanitaire participe de cet objectif en incitant les personnes déplacées à demeurer dans leur pays d’origine ou dans les pays limitrophes. Ceci a stimulé une augmentation des budgets d'aide humanitaire, mais aussi une affectation croissante des fonds ${ }^{12}$ qui limite l'autonomie et la flexibilité des organisations et accroît le contrôle des donateurs. Cette conclusion nous intéresse puisqu'elle pourrait expliquer, au moins partiellement, l'augmentation et l'évolution de la nature du financement au HCR. Alors en effet que les politiques d'asile du Canada se durcissent ${ }^{13}$, le pays se montre de plus en plus généreux envers le HCR. Ce qui à première vue peut semble paradoxal pourrait s'inscrire dans une logique de maintien des populations loin des frontières nationales du pays, par le biais du HCR.

Le soutien canadien au HCR s'inscrit dans un cadre humanitaire plus large dont l'objectif est de «sauver des vies, alléger la souffrance et [à] protéger la dignité des personnes touchées par les conflits et les catastrophes naturelles grâce à des interventions appropriées, efficaces et rapides ${ }^{14}$, en respectant les principes humanitaires d'humanité, d'impartialité, de neutralité et d'indépendance. Il a toutefois été documenté qu'au-delà des considérations purement humanitaires, la politique d'aide du Canada sert des intérêts commerciaux ${ }^{15}$, sécuritaires ${ }^{16}$, idéologiques ${ }^{17}$ et de politique domestique ${ }^{18}$, à l'instar de celle d'autres pays donateurs. Si la politique d'aide internationale fait l'objet d'une vaste littérature, les orientations de la politique publique d'aide humanitaire du Canada sont moins bien documentées. Néanmoins, en matière de politique publique, la distinction entre l'aide d'urgence et l'aide au développement semble caduque. Les scénarios dans lesquels sont menées les opérations se sont en effet considérablement complexifiés et ces interventions s'inscrivent la plupart du temps dans la durée, requérant successivement ou en alternance des projets d'urgence, de réhabilitation/ reconstruction et de développement. Par ailleurs, au niveau opérationnel, la division entre aide au développement et aide humanitaire est inappropriée, l'aide étant dans les deux cas administrée au moins en partie par les mêmes acteurs et financée par les mêmes bailleurs étatiques. Ainsi, même si les logiques d'action diffèrent, les politiques d'aide humanitaire du Canada font partie de la politique publique d'aide internationale.

Les politiques d'aide internationale ont été largement transformées dans les dernières années. La «guerre au terrorisme» a engendré un changement de paradigme tant sur le plan discursif que pratique, et ce dans la vaste majorité des pays occidentaux dont le Canada ${ }^{19}$. Le principal argument repose sur une prémisse simpliste où la pauvreté serait le catalyseur des conflits violents, lesquels deviennent des lieux de recrutement de terroristes. Laide internationale est de ce fait envisagée à travers le prisme de la sécurité nationale et comme un outil parmi d'autres pour lutter contre le terrorisme et l'insécurité. En dépit des critiques de nombreux chercheurs quant au bien-fondé de cette approche, elle se trouve au centre $\mathrm{du}$ "tout terrorisme $»^{20}$. Les pays occidentaux ont 
ainsi embrassé le principe de l'aide au développement et de l'assistance aux réfugiés comme vecteurs de sécurité. Cette grille d'analyse alimente notre réflexion.

Cet article présente une étude de cas préliminaire sur le financement canadien au HCR et, plus largement, contribue au développement de la recherche sur le financement humanitaire. La recherche examine si l'augmentation de la contribution canadienne au HCR peut être comprise comme un prolongement des politiques nationales d'asile et de la volonté de maintenir à l'écart des populations jugées indésirables, ou encore de la politique étrangère (qui devrait être cohérente avec la politique intérieure). Létude explore également si cette contribution correspond aux priorités du HCR ou témoigne d'un manque de confiance envers l'organisation. Enfin, la recherche s'interroge sur l'influence que peut exercer, à travers son financement, le Canada sur le HCR.

Dans ce dessein, après une brève section méthodologique, nous présentons les principales données de financement, puis nous les analysons à la lumière des priorités canadiennes de développement établies par le ministère des Affaires étrangères, du Commerce et du Développement (MAECD), de l'évolution de la politique nationale d'immigration et d'asile et des orientations du HCR. Au-delà du fait que le financement canadien suit une tendance internationale pour répondre aux besoins humanitaires grandissants de populations réfugiées, nous observons que le financement au HCR participe de la politique intérieure et extérieure canadienne en visant des pays qui sont à la fois les lieux d'origine ou les premiers pays d'accueil de réfugiés et ont été définis comme des cibles privilégiées de l'aide internationale canadienne, tout en répondant à des impératifs humanitaires. Nous remarquons également qu'une meilleure compréhension des processus de prise de décision et du rôle des émotions dans ce processus serait nécessaire pour affiner notre analyse. Enfin, l'influence canadienne sur le HCR à travers son financement ne peut quêtre relativement limitée, bien que la constance du pays puisse le doter d'une certaine autorité morale.

\section{Méthodologie}

Notre étude est basée sur l'analyse de données financière du MAECD et du HCR, et la consultation de documents officiels pertinents. Quelques personnes clés ont été rencontrées de façon officieuse. Ces personnes souhaitant demeurer anonymes, elles ne sont pas directement citées, même si ces discussions ont enrichi l'analyse. Pour étudier notre question de recherche, nous comparons tout d'abord spécifiquement lévolution du financement canadien au HCR et à deux autres agences importantes de l'onu, le Programme alimentaire mondial (PAM) et le Fonds des Nations Unies pour l'enfance (UNICEF). Afin d'approfondir notre examen, nous comparons ce financement à l'aide multilatérale et à l'aide internationale en général. Dans un second temps, nous nous penchons sur l'évolution de l'aide affectée canadienne au HCR et la comparons à celle de l'aide affectée par l'ensemble des donateurs du HCR. Cela nous permet de comprendre si le Canada suit une tendance générale ou s'il tend à agir de manière autonome. Nous étudions ensuite à quels pays est destinée l'aide affectée du Canada afin de tenter de déterminer ce qui influence la sélection de ces pays. Pour vérifier si les appels de fonds du HCR motivent les décisions de financement, nous examinons si ces pays correspondent à ceux qui sont ciblés en priorités par le HCR ou qui ont fait l'objet d'appels d'urgence. Ceci ne s'avérant pas concluant, nous explorons si un lien peut être fait avec les pays ciblés par l'aide internationale du Canada ou encore les principaux pays d’origine des réfugiés au Canada.

Nous avons combiné plusieurs sources afin de mieux saisir différentes facettes du financement canadien au HCR. Notre source d'information principale pour l'ensemble du financement du pays au HCR, à l'UNICEF, au PAM et à l'ONU en général est une réponse détaillée du ministre des Affaires étrangères, John Baird, à une demande de renseignement faite au gouvernement par la députée de Laurier/SainteMarie, Hélène Laverdière et concernant le financement et la participation du Canada au sein de l'oNu ${ }^{21}$. Les données sur l'aide multilatérale et bilatérale sont tirées des rapports statistiques annuels sur l'aide internationale de l'Agence canadienne de développement international (ACDI) jusqu'en 2012-13 ${ }^{22}$. Le rapport 2013-14 a été préparé par le ministère des Affaires étrangères, du Commerce et du Développement (MAECD) au sein duquel ont été fusionné l'ACDI et le ministère des Affaires étrangères et du commerce international en $2013^{23}$. Pour le financement affecté à une aire géographique, à un thème ou à un secteur, les sources d'information principales sont les projets du HCR inclus dans la banque de projets de développement international du MAECD en ligne ${ }^{24}$, ainsi que les profils des donateurs dressés par le HCR dans ses rapports annuels ${ }^{25}$ et ses appels globaux et d'urgence. Enfin, les statistiques sur l'origine des réfugiés au Canada ont été compilées à notre demande par Citoyenneté et Immigration Canada $^{26}$. Les données sur l'évolution de l'aide canadienne au HCR et sur le financement affecté sont principalement présentées sous forme de graphiques, puis sont réexaminées et analysées dans la section suivante. Le choix de la période analysée s'explique par la disponibilité des données.

Une précaution méthodologique quant à l'exactitude des chiffres s'impose. D'abord, il n'est pas possible de faire coïncider les données canadiennes et celles du HCR, les années financières considérées étant en léger décalage (celle du gouvernement canadien débute le ier avril et se termine le 31 mars alors que celle du HCR correspond à une année calendaire). Lévolution du financement et les montants rapportés 


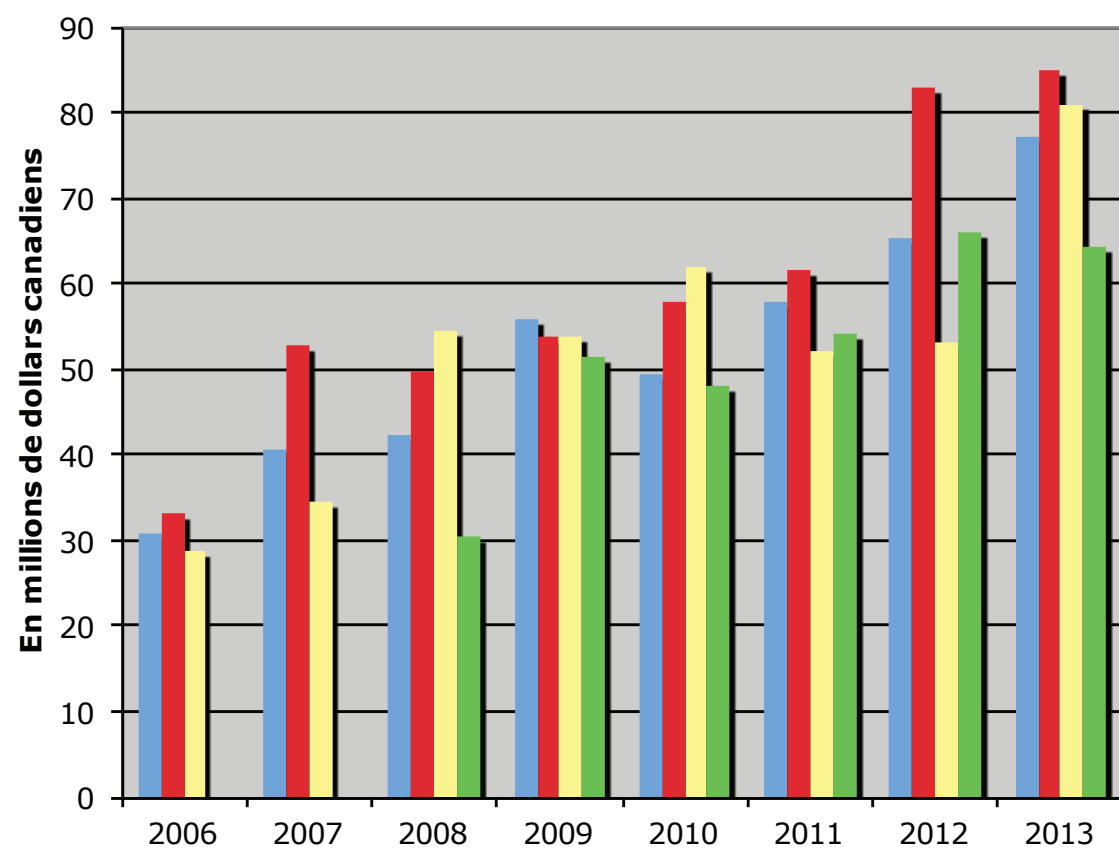

IHCR

MAECD*

FTS UN-OCHA**

- Banque de projets MAECD

Figure 1: Comparaison des données du financement canadien au HCR, 2006-2013, selon diverses sources

Sources: Profils des donateurs du HCR (2007-2014); réponse à la question Q-598, ministre des Affaires étrangères, 2014; Financial Trac-

king Service, оснA; Banque de projets de développement international, Affaires mondiales Canada

* Réponse à la question Q-598, ministre des Affaires étrangères, 2014

${ }^{* *}$ Convertis en dollars canadiens, selon l'année de référence (http://www.oanda.com/lang/fr/currency/converter).

par les deux institutions sont néanmoins similaires. Ensuite, lorsqu'il s'agit d'étudier le financement affecté, les montants obtenus en additionnant les projets du HCR inclus dans la banque du gouvernement à compter de 2009 ne coïncident pas parfaitement avec les montants globaux fournis par le gouvernement et le HCR. Aussi, les chiffres sur le financement humanitaire compilés par le Bureau de coordination des affaires humanitaires des Nations Unies dans son Financial Tracking Service (FTS) ${ }^{27}$ présentent des différences avec les trois autres sources de données (fig. 1). Les démarches faites auprès du gouvernement canadien ne nous ont pas permis de clarifier ces données. Dans tous les cas, les tendances sont toutefois semblables et, à cet égard, les différences entre les données issues de diverses sources n'invalident pas nos observations.

Nous avons choisi d'utiliser la banque de projets du gouvernement canadien plutôt que celle du FTs pour procéder à l'analyse des financements affectés, car nous avons présumé de l'exactitude des données rendues publiques par le gouvernement. Il faut néanmoins noter que leur présentation sur le site gouvernemental induit en erreur en suggérant que les financements régionaux sont affectés de façon précise à des pays donnés ce qui, aux dires des fonctionnaires, est inexact. Les données compilées par le FTs sont fournies sur une base volontaire par les pays donateurs et les organisations bénéficiaires. Certaines contributions ne sont de ce fait pas rapportées. Par ailleurs, le FTs contient des erreurs manifestes: des financements sont inclus à plus d'une reprise tandis que d'autres non confirmés sont inclus aux données.

\section{Présentation des données: le financement canadien au HCR}

Cette section présente les principales données de financement au HCR. Nous nous intéressons dans un premier temps à l'évolution de l'aide internationale canadienne et, plus spécifiquement, à celle de la contribution au HCR. Nous explorons ensuite la nature de l'aide affectée et les aires géographiques ciblées. Nos observations sont généralement replacées dans un contexte international afin de déterminer si la tendance canadienne correspond à une tendance plus générale. 


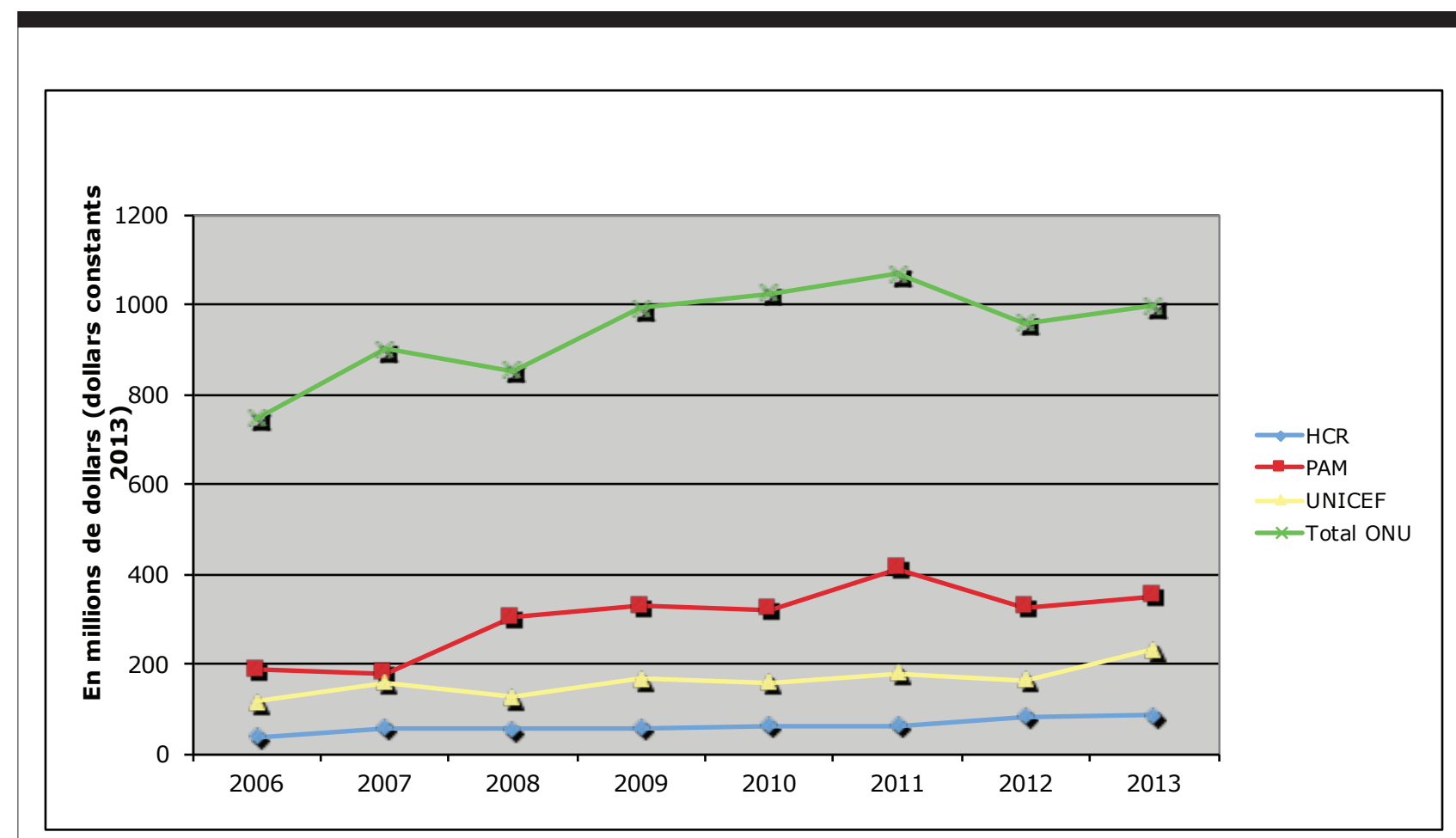

Figure 2: Financement canadien aux Nations Unies, 2006-2013

Source: Réponse à la question Q-598, ministre des Affaires étrangères, 2014

Évolution de l'aide internationale canadienne, 2006-2013

Entre 2006 et 2013, l'aide canadienne au HCR augmente de $128 \%$. Elle est donc multipliée par 2,28. Le financement à l'UNICEF croit pour sa part de $102 \%$ et celui au PAM de $88 \%$ (fig. 2). Bien que ces agences des Nations Unies aient des mandats différents, et que les activités du PAM et de l'UNICEF ne soient pas strictement humanitaires, la comparaison nous semble pertinente afin de mettre en évidence la croissance du financement du HCR, mais également son importance relative par rapport à d'autres agences onusiennes ayant au moins en partie une vocation humanitaire. Si l'augmentation du financement au HCR est en effet plus importante, la valeur réelle de cette contribution demeure considérablement inférieure à celle du PAM ou de l'Unicef. Par exemple, en 2013, plus du tiers du financement canadien de 997,2 millions à l'ONU est donc destiné au PAM (contre un quart en 2006), près du quart à l'UNICEF (15\% en 2006) et moins de $10 \%$ au HCR ( $5 \%$ en 2006). Durant cette période, le financement à l'ensemble de l'onu ne croit que de $34 \%$.

La proportion de laide multilatérale canadienne allouée à l'ensemble des agences spécialisées, institutions et organes de l'Organisation des Nations Unies demeure relativement stable, variant entre 61 et $78 \%$ (fig. 3). Par conséquent, si le financement du PAM, de l'UNICEF et du HCR connait une croissance importante, d'autres agences d'envergure voient leur financement diminuer durant le même intervalle, dont le Programme des Nations Unies pour le développement (137,1 à 96 millions) et le Fonds des Nations Unies pour la population (53,4 à 41 millions).

Entre 2006 et 2013, le budget annuel du HCR augmente significativement en raison de crises qui ont entraîné d'importants déplacements de population, notamment en Somalie, en Syrie et en République Démocratique du Congo (RDC). Par conséquent, même si la contribution canadienne au HCR croît, la part du budget de l'agence fournie par le Canada diminue de $2,3 \%$ en 2006 à 1,6\% en 2013. Ainsi, la contribution dautres donateurs importants augmente elle aussi considérablement durant cette période (fig. 4). En fait, la contribution des trois pays donateurs les plus importants connaît une croissance encore plus marquée que celle du Canada - les États-Unis, le Japon et la Commission européenne (CE) fournissent en effet la moitié environ du budget annuel du HCR (sauf en 2006 où la Suède a donné davantage que l'Europe). À eux seuls, les États-Unis couvrent environ le tiers du budget de l'agence, alors que le Japon et la CE en financent moins d'un dixième chacun ${ }^{28}$. Leurs contributions respectives augmentent de $182 \%$, 200\% et $139 \%$ entre 2006 et 2013. Au cours de cette période, la contribution de l'Allemagne croît de façon encore plus importante ( $234 \%$ ), alors que celle des Pays-Bas et des pays scandinaves s'apprécie plus 


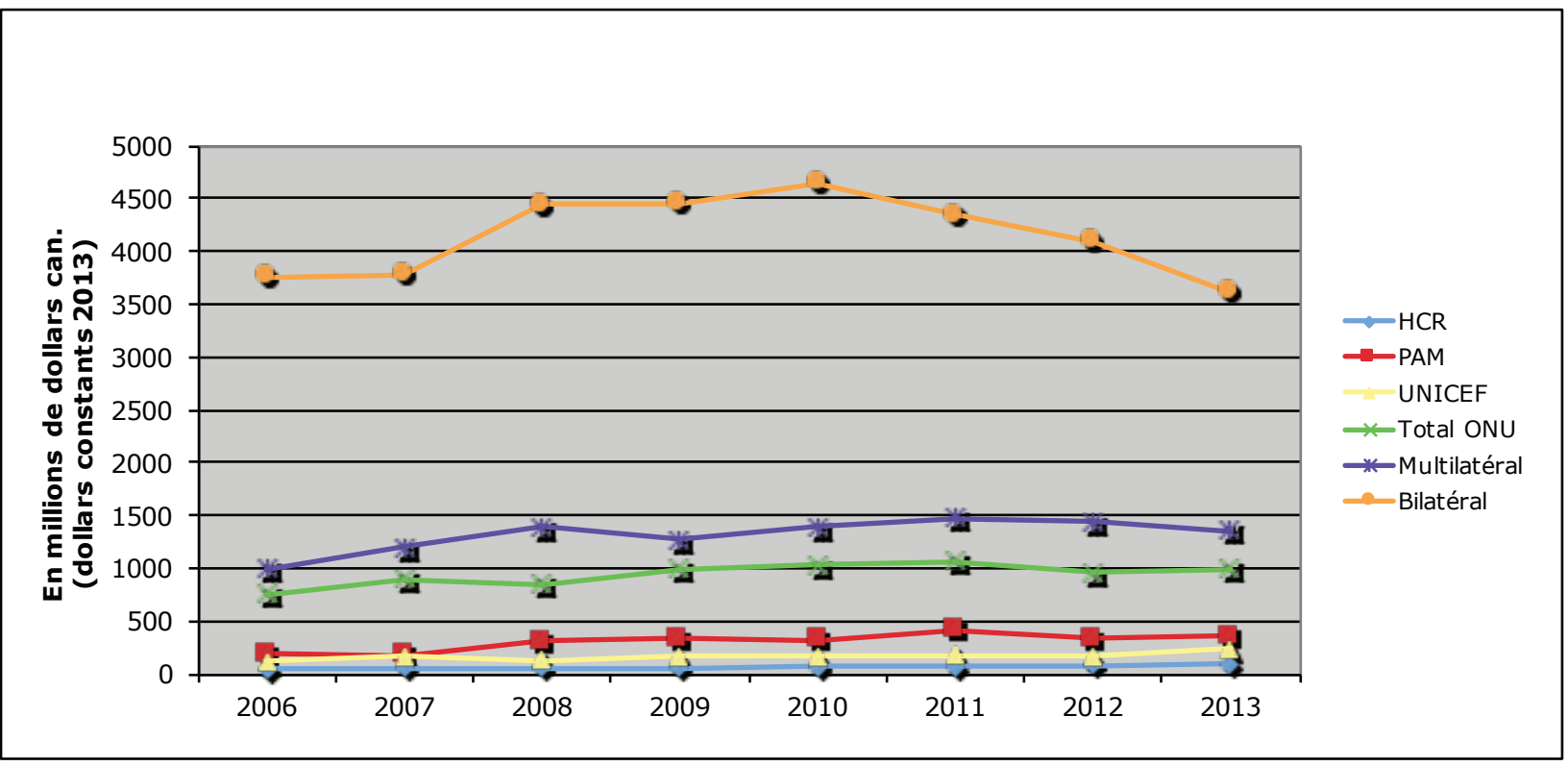

Figure 3: Aide internationale canadienne, 2006-2013

Sources: Rapports statistiques annuels de l'ACDI (ACDI 2009-2014) et du MAECD (2015); réponse à la question Q-598, ministre des Affaires étrangères, 2014

lentement (14\% pour les Pays-Bas, $29 \%$ pour la Norvège, $51 \%$ pour le Danemark et $69 \%$ pour la Suède). Le financement du Koweït, un pays ne comptant parmi les donateurs importants qu'à partir de 2013, augmente de $1419 \%$. Si l'on exclut le Koweït du calcul, le financement Canadien s'est apprécié légèrement plus rapidement que celui de la moyenne des principaux donateurs mais, en valeur réelle, demeure l'un des plus bas de ce groupe (11e en 2006, 2008 et 2013, 9e en 2007,2009 et 2012 , 8e en 2011, 10e en 2010).

\section{Évolution de l'aide affectée canadienne}

Entre 2006 et 2013, les contributions du Canada au HCR sont de plus en plus ciblées, le financement non affecté diminuant de 45 à $17 \%$. En valeur nominale, l'appui au fonctionnement institutionnel du HCR demeure stable, sétablissant à 14 millions de dollars (ce qui représente une diminution de 1,2 millions en valeur réelle entre 2006 et 2013), alors que le financement affecté augmente. En 2014, le financement institutionnel diminue à 12,6 millions. En 2006, la part du financement non affecté à l'ensemble des Nations Unies est considérablement moindre que celle du HCR mais, en 2013, ces niveaux sont comparables (fig. 5).

Une telle réduction de la proportion des fonds non affectés est une tendance observable chez plusieurs autres donateurs importants, à l'exception de la Suède et de la Norvège qui ont plutôt considérablement délié leur aide au cours de cette période, et des États-Unis et de la CE dont l'aide ne pouvait être affectée davantage, celle-ci leétant déjà entièrement (fig. 6) ${ }^{29}$. Environ un dixième du financement japonais n'est pour sa part plus affecté depuis 2007. Jusqu'alors, ce pays affectait également l'entièreté de sa contribution au HCR. La proportion non affectée de l'aide canadienne est légèrement supérieure à la moyenne, mais cet écart s'estompe rapidement. En 2006, alors que $20 \%$ des contributions globales nétaient pas affectées, cétait le cas de $45 \%$ de la contribution canadienne. En 2013, la différence est négligeable: $16 \%$ des contributions globales ne sont pas affectées, contre $17 \%$ de la contribution canadienne ${ }^{30}$.

\section{Cibles du financement affecté}

Les données sur le financement affecté entre 2010-2012, tirées de la banque de projets gouvernementaux, permettent d'identifier les pays visés d'une année à l'autre. Ils sont neufs en 2010, 19 en 2011 et 15 en 2012. Sept pays se retrouvent systématiquement parmi les dix principaux pays ciblés, soit le Pakistan, le Soudan (du Sud à partir de 2012), la RDC, la Colombie, l'Irak, le Kenya et le Tchad. L'Éthiopie est pour sa part présente en 2011 et 2012. Le choix de ces pays et des aires géographiques ne coïncide que partiellement avec les priorités du HCR. En 2010, par exemple, alors que le HCR identifie 


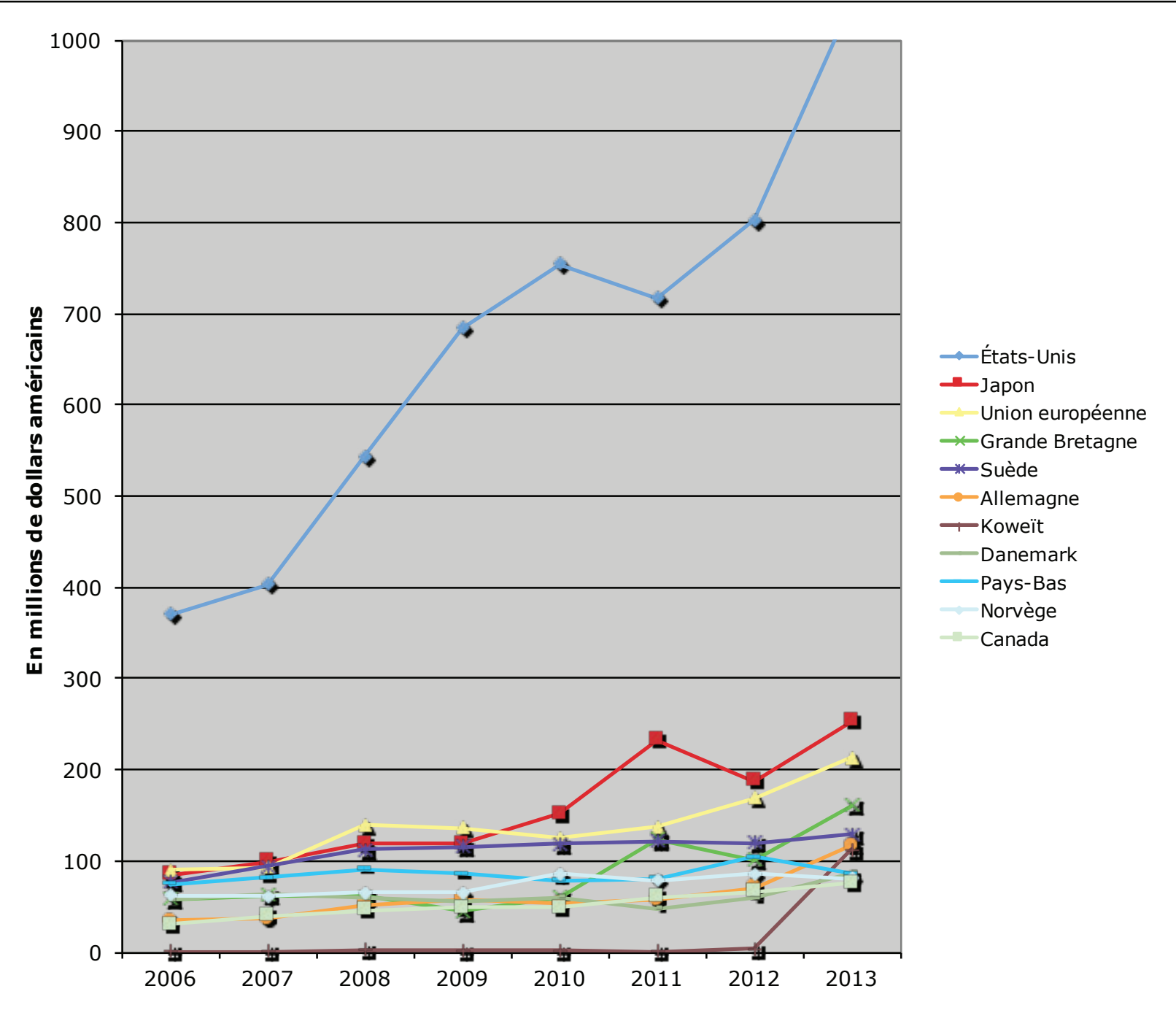

Figure 4: Évolution du financement des dix principaux donateurs de 2013, de 2006 à 2013

Sources: Profils des donateurs du HCR (2007-2014)

dans l'ordre l'Irak, le Pakistan et le Soudan comme pays prioritaires $^{31}$, le Canada cible le Soudan et le Tchad (ensemble), le Pakistan et la République Démocratique du Congo. L'Irak arrive en quatrième position. Ces différences pourraient s'expliquer par la réponse à des appels supplémentaires. Cette même année, la proportion du financement canadien attribué à diverses régions ne correspond pas à celles qui ont été établies par le HCR dans son appel global. Le HCR précise que les fonds nécessaires pour ses opérations en Afrique mobilisent la part la plus importante de son budget. Les régions du Moyen-Orient et de l'Afrique du Nord, de l'Asie et du Pacifique, de l'Europe et des Amériques suivent, dans l'ordre. Or, la contribution canadienne, tant en considérant l'ensemble du financement affecté à un pays ou une (sous)-région qu'en ne considérant que le financement (sous)-régional, ne respecte cet ordre que pour l'Afrique, région qui reçoit la part la plus importante du financement. L'Asie et Pacifique devancent le Moyen-Orient et l'Afrique du Nord, régions suivies par les Amériques. Aucun financement n'est accordé à l'Europe. Ainsi, les fonds ne sont pas strictement répartis selon les priorités géographiques établies par le HCR.

Une part importante des fonds affectés l'est en réponse à des appels supplémentaires et des plans d'action du HCR, ou encore d'un appel de fonds consolidé coordonné par 


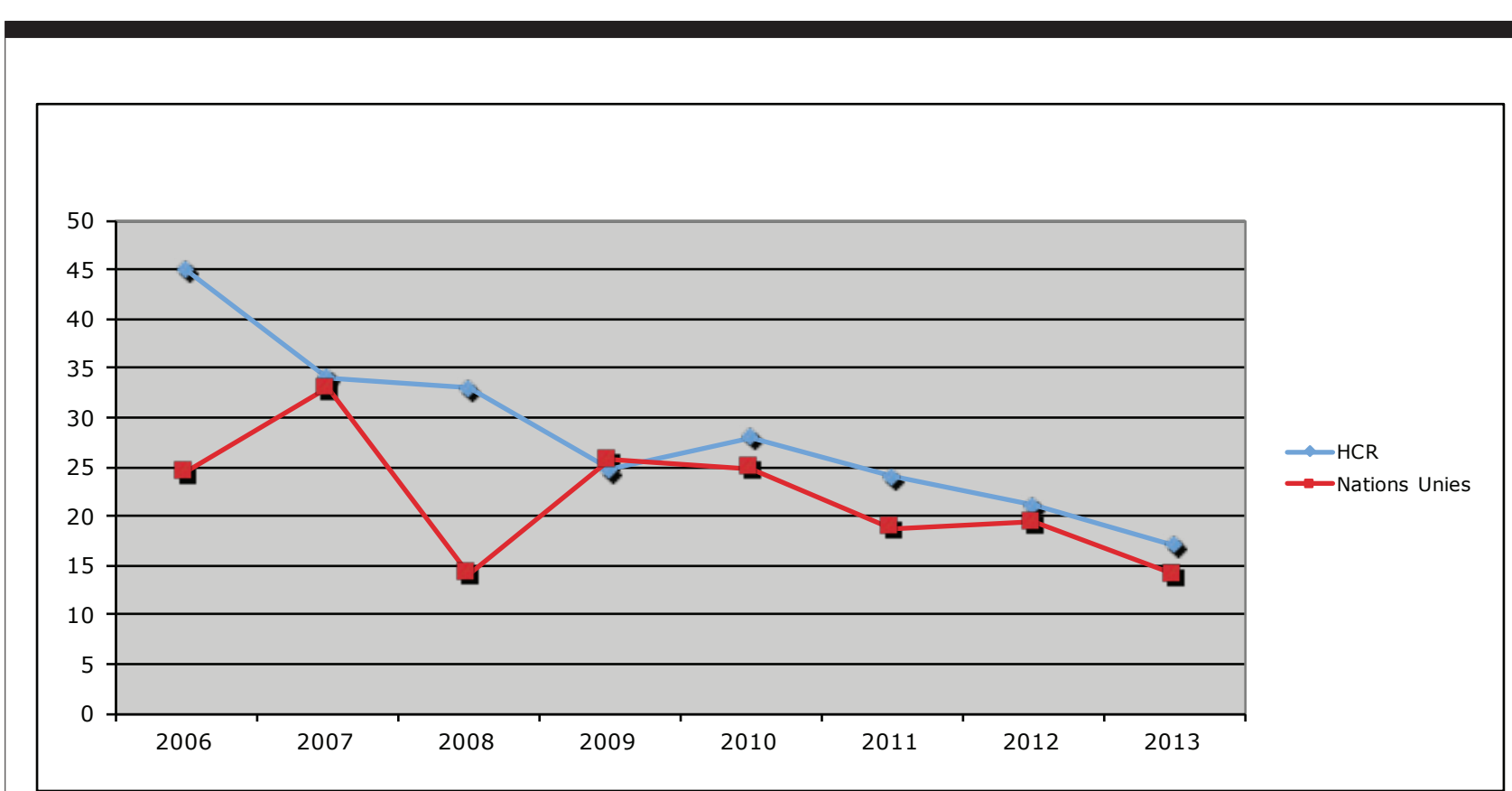

Figure 5: Proportion du financement canadien non affecté, 2006-2013

Sources: Profils des donateurs du HCR (2007-2014); Réponse à la question Q-598, ministre des Affaires étrangères, 2014

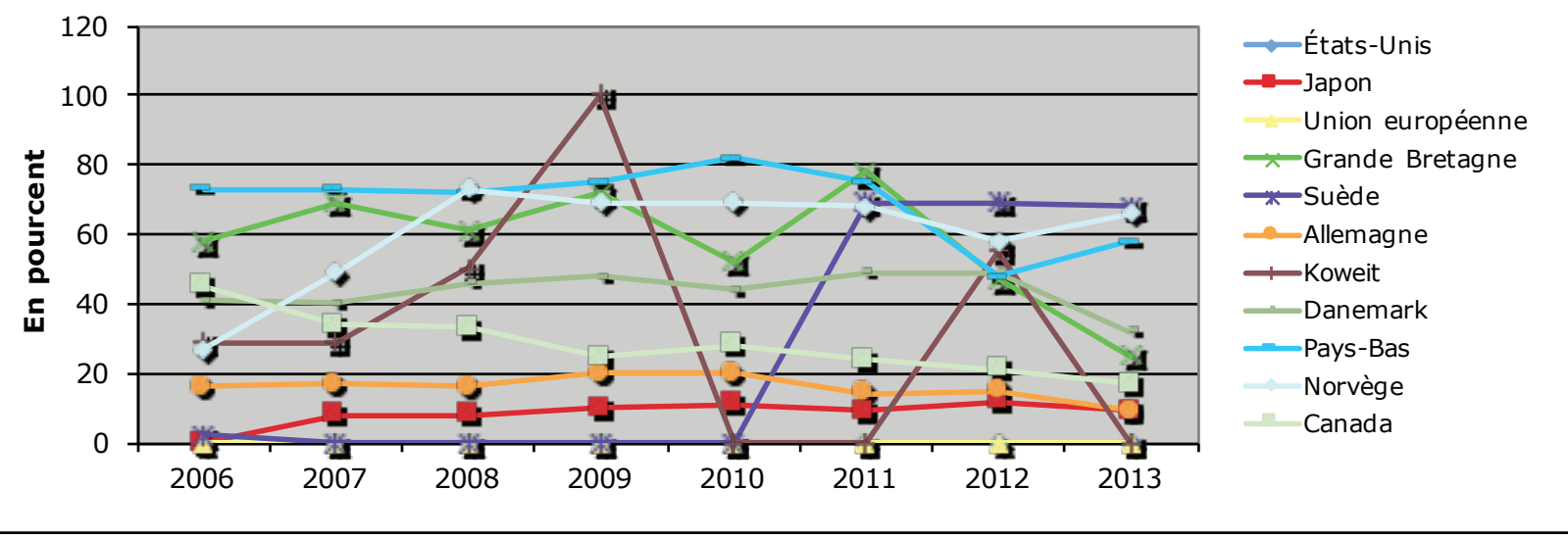

Figure 6: Proportion du financement non affecté au HCR, 2006-2013

Sources: Profils des donateurs du HCR (2007-2014)

le Bureau de coordination des affaires humanitaires des Nations Unies. Ceci lie le financement à des interventions particulières comme une opération de rapatriement, un déplacement lié à un conflit ou une catastrophe naturelle, ou encore une réponse régionale pour une situation de déplacement à grande échelle. Or, les appels spéciaux ne sont pas tous financés et certains fonds visant un pays en particulier ne sont pas liés à un appel spécial. Par exemple, en 2011, le Canada n'a vraisemblablement ${ }^{32}$ pas répondu aux appels d'urgence pour les réfugiés soudanais en Éthiopie, pour le rapatriement des réfugiés angolais ou encore pour le Kirghizstan. Bien qu'aucun appel d'urgence ne semble avoir été émis, le pays a affecté des fonds spécifiques à l'Ouganda, au Bangladesh et au Népal.

(C) Catherine-Lune Grayson et François Audet, 2017. Cette œuvre en libre accès fait l'objet d'une licence Creative Commons Attribution-NonCommercial 4.0 International License, laquelle autorise l'utilisation, la reproduction et la distribution de l'œuvre sur tout support à des fins non commerciales, pourvu que l'auteur ou les auteurs originaux soient mentionnés et que la publication originale dans Refuge : revue canadienne sur les réfugiés soit citée. 


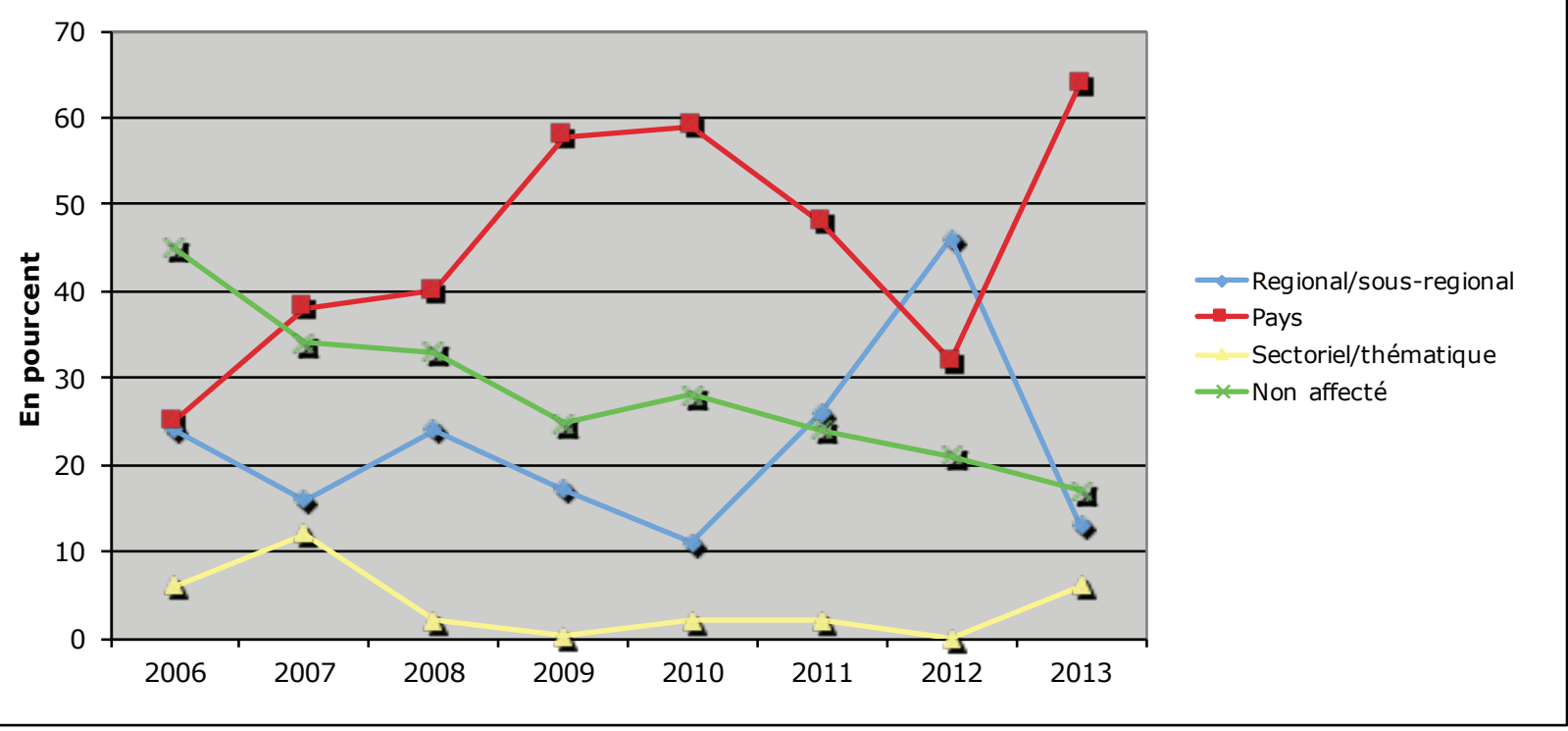

Figure 7: Évolution du financement affecté canadien au HCR, 2006-2013 (proportions)

Source: Banque de projets de développement international, Affaires mondiales Canada

\section{Discussion}

Les données de la section précédente montrent que le financement canadien au HCR ne coïncide pas parfaitement avec les priorités exprimées par cette même agence. Il est donc raisonnable d'affirmer que les choix canadiens ne peuvent être expliqués entièrement par les demandes du HCR qui devraient correspondre aux priorités internationales en matière d'assistance et de protection des personnes déplacées. Cela nous incite à nous attarder aux pays ciblés par l'aide et à tenter d'interpréter les raisons qui sous-tendent ces choix, afin de mieux cerner les priorités politiques et humanitaires du gouvernement canadien.

Nous explorons pour ce faire trois questions complémentaires. Bien qu'il soit censé être principalement motivé par des considérations humanitaires et non de politique extérieure, le financement humanitaire fait au HCR peut-il être compris comme un prolongement de la politique étrangère? Peut-on plutôt y voir un prolongement de la politique nationale d'immigration et d'asile, tout en considérant que ces deux axes politiques ne sont pas mutuellement exclusifs, au contraire? Enfin, le financement affecté peut-il être interprété comme un manque de confiance envers le HCR?

Avant d'étudier ces trois questions, il convient de souligner la complexité des processus décisionnels dans les politiques publiques, processus qui demeurent mal documentés. Le récent rapport du Vérificateur général du Canada observe d'ailleurs que les financements humanitaires du gouvernement sont liés aux besoins des populations, mais que les processus décisionnels sont flous. Ainsi, les raisons qui justifient la répartition de laide entre diverses crises et divers projets ne sont pas expliquées ${ }^{33}$. Il est probable que plusieurs facteurs influencent la prise de décision, dont la sensibilité et l'intérêt particulier des fonctionnaires ${ }^{34}$. Hassenteufel écrit que la prise de décision découle d'interactions entre de multiples acteurs qui s'influencent mutuellement ${ }^{35}$. Dans le cas d'une crise humanitaire, le plaidoyer des organisations humanitaires, le travail des médias ${ }^{36}$, la proximité géographique et les intérêts étatiques ${ }^{37}$, ainsi que les valeurs des administrateurs publics ${ }^{38}$ sont susceptibles d'influencer l'importance accordée à la crise.

\section{Un prolongement de la politique extérieure?}

En 2009, le Canada a énoncé ses priorités de développement international dans son Plan d'action sur l'efficacité de l'aide, un engagement réitéré en 2014. Ce plan formule quatre grands objectifs: établir des partenariats efficaces, cibler les efforts sur les plans géographique et thématique, produire des résultats et les maintenir, et accroître la transparence et la responsabilisation. Le Canada inclut l'onu parmi ses partenaires prioritaires identifiés sous le premier objectif, tout comme les organisations de la société civile, les partenaires privés, la diaspora au Canada, et la communauté scientifique

(C) Catherine-Lune Grayson et François Audet, 2017. Cette œuvre en libre accès fait l'objet d'une licence Creative Commons Attribution-NonCommercial 4.0 International License, laquelle autorise l'utilisation, la reproduction et la distribution de l'œuvre sur tout support à des fins non commerciales, pourvu que l'auteur ou les auteurs originaux soient mentionnés et que la publication originale dans Refuge : revue canadienne sur les réfugiés soit citée. 
et technologique canadienne. Le deuxième objectif identifie les pays privilégiés par l'aide bilatérale canadienne, pays choisis en fonction de leurs besoins, mais également de leur «alignement sur les priorités canadiennes et leur capacité à utiliser l'aide efficacement ${ }^{39}$. En 2009 , le Canada avait annoncé qu'il consacrerait $80 \%$ de ses ressources bilatérales à 20 pays en développement ciblés ${ }^{40}$. Ce nombre passe à 25 en 2014 et 12 partenaires prioritaires du développement sont identifiés. Ce dernier objectif définit les thèmes prioritaires du gouvernement: accroître la sécurité alimentaire, assurer l'avenir des enfants et des jeunes, favoriser une croissance économique durable, promouvoir la démocratie, ainsi que la stabilité et la sécurité. Le gouvernement souligne également sa volonté de répondre rapidement aux crises humanitaires.

Laugmentation du financement au HCR ne semble pas s'inscrire dans l'un des axes thématiques de la politique de développement canadienne, à moins de considérer que ce financement participe aux objectifs de stabilité et de sécurité, mais il pourrait témoigner de l'engagement du gouvernement à répondre aux crises humanitaires. Cette impression doit néanmoins être examinée de façon critique puisque les priorités définies par le HCR norientent pas totalement le financement canadien. Laugmentation du financement du PAM et de l'UNICEF peut pour sa part être attribuée à deux des priorités thématiques, soit contribuer à la sécurité alimentaire et assurer l'avenir des enfants et des jeunes.

Les pays ciblés par l'aide affectée au HCR correspondent au moins en partie à ceux qui sont ciblés par l'aide bilatérale. En effet, trois des pays qui comptent parmi les principaux bénéficiaires de l'aide affectée du Canada au HCR comptent parmi les pays ciblés plus largement par les politiques canadiennes en 2009, soit le Pakistan, le Soudan et la Colombie. Tous à l'exception du Tchad font partie de la nouvelle liste publiée en 2014, à titre de pays ciblés ou de partenaires du développement. Or, et ceci sera également vrai lorsque nous discuterons de la relation entre les pays d'origine des réfugiés au Canada et le financement au HCR, il s'agit également de pays dont une partie de la population a été déplacée, ou encore de premiers pays d'accueil de réfugiés. Il est dès lors impossible d’affirmer que le choix de ces pays est strictement lié à des objectifs de politique extérieure et non à des considérations humanitaires.

Dans son Plan d'action sur l'efficacité de l'aide, le MAECD précise que le «fait de travailler avec [des partenaires multilatéraux et mondiaux] permet aussi au Canada d'orienter l'élaboration du programme de développement international». De la même façon, l'un des objectifs du ministère de la Citoyenneté et de l'Immigration est d'influencer «les objectifs stratégiques internationaux en matière de mouvements migratoires et d'intégration ${ }^{41}$. La croissance du financement au HCR, qui permet au Canada de demeurer parmi les donateurs importants de l'agence, pourrait témoigner de la volonté de conserver une influence sur l'organisation et de s'assurer que ses interventions correspondent aux objectifs politiques canadiens. Or, la contribution canadienne ne représentant qu'une petite part du financement global du HCR, l'influence du Canada sur l'organisation, du moins par son financement, ne peut être que relativement limitée. À l'inverse, les États-Unis exercent à cet égard une influence considérable, notamment sur les programmes et l'embauche $\mathrm{du}$ personnel ${ }^{42}$. Lon peut donc présumer que le Canada se reconnait globalement dans les objectifs du HCR ou encore que son financement participe plus largement de ses orientations politiques, y compris humanitaires. En fait, si l'influence strictement financière du Canada sur le HCR est sans doute relativement limitée, la régularité de son engagement lui donne éventuellement une certaine autorité morale, contribuant à renforcer certaines de ses positions et à rallier le support d'autres États. Par exemple, le pays souligne qu'il s'intéresse particulièrement à la recherche de solutions quant aux situations de déplacement prolongées, ce qui s'est traduit par une contribution de trois millions en 2014-2015 pour soutenir la stratégie globale du HCR dans ce domaine ${ }^{43}$.

En somme, le financement du Canada au HCR pourrait, jusquà un certain point, s'inscrire dans les priorités de développement du pays. Nous ne pouvons cependant attribuer la croissance et la nature du financement strictement à des considérations de politique extérieure. Aussi, étant donné la proportion que représente le financement canadien sur le budget total du HCR, l'influence financière du Canada semble négligeable.

\section{Le financement au HCR: un prolongement des politiques} nationales d'asile?

Le HCR oeuvrant auprès des populations déplacées et réfugiées, il est pertinent de se s'interroger sur la relation entre le financement du Canada au HCR et les politiques migratoires et d'asile du pays. Pendant que la contribution canadienne au HCR augmente, de 2006 à 2013, le nombre de nouveaux réfugiés admis au Canada diminue (fig. 8). Le gouvernement manifeste également publiquement sa volonté de restreindre l'accès à l'asile, une rhétorique motivée par la protection des Canadiens et du territoire, une tendance observable dans plusieurs pays occidentaux 44 . En 2011, le ministère de la Citoyenneté et de l'Immigration précise que son intervention a notamment pour objectif de contribuer à :

la capacité de la communauté internationale de gérer ces pressions migratoires qui conduisent des gens à nos portes, dans le but de préserver l'intégrité et d’assurer la viabilité de nos propres programmes d'immigration et de protection des réfugiés ${ }^{45}$.

Le désir de limiter l'accès au territoire canadien de potentiels demandeurs d'asile se traduit par des mesures de dissuasion, 


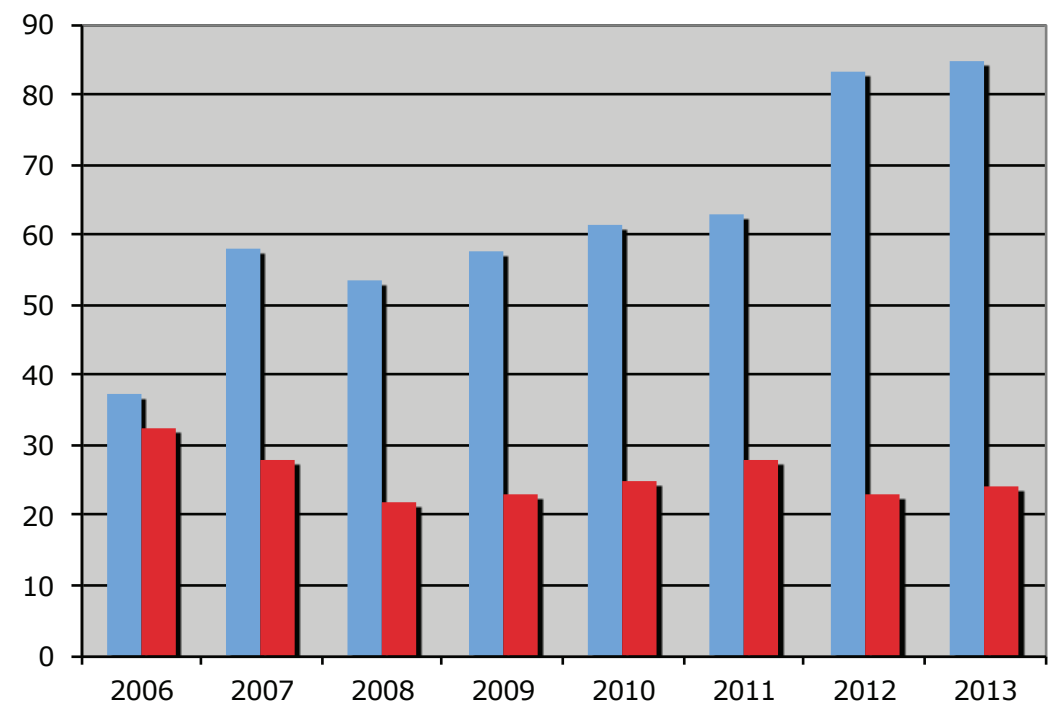

Financement can HCR (en milions de dollars constants 2013)

- Réfugiés au Canada (en milliers)

Figure 8: Réfugiés au Canada et financement canadien au HCR, 2006-2013

Sources: Réponse à la question Q-598, ministre des Affaires étrangères, 2014; «Faits et chiffres 2013 - Aperçu de l’immigration: Résidents permanents.» Citoyenneté et Immigration Canada

d'externalisation et d'interception exigeant, entre autres, que des gens originaires de pays sources de réfugiés, dont l'Afghanistan, la Colombie, l'Irak, la Somalie ou le Soudan du Sud, obtiennent des visas, ou encore imposant de lourdes peines financières aux transporteurs accusés d'avoir pris des migrants irréguliers à bord ${ }^{46}$. Les changements apportés à la Loi sur l'immigration et la protection des réfugiés en décembre 2012 s'inscrivent également en ce sens en imposant des délais très courts aux demandeurs d'asile, en limitant les droits d'appel de demandeurs originaires de pays désignés «sûrs» (une mesure jugée inconstitutionnelle par la Cour fédérale du Canada en $2015^{47}$ ) ou encore en réduisant l'accès à certains services ${ }^{48}$.

Quelques auteurs ont déjà souligné que les États affectent, entre autres, leur financement sur la base de préoccupation migratoires 49 On peut se demander si, comme le suggèrent Bermeo et Leblang50, plus les États souhaitent contrôler l'arrivée de migrants et de réfugiés, plus ils sont enclins à accroître leur aide à ce pays, notamment à travers des organisations internationales, dans l'espoir que l'aide humanitaire sur place contribue à limiter les mouvements et les arrivées. Les choix de financements canadiens (et la politique d'aide au développement) pourraient ainsi être influencés par les pays d'origine des plus grands groupes de réfugiés arrivés au Canada ces dernières années soit, de 2006 à 2010, la Colombie,
l'Irak, l'Afghanistan, la Chine, le Sri Lanka, le Myanmar, la RDC, l'Éthiopie, la Somalie et le Pakistan ${ }^{51}$. (En considérant la période 2006-2013, la liste change très légèrement: s'y ajoutent Haïti et l'Érythrée, alors que le Pakistan et le Myanmar en sont retranchés.) Les principaux pays ciblés par l'aide affectée du Canada au HCR dans les années qui suivent, soit en 2011 et 2012, incluent plusieurs de ces pays (Colombie, Irak, RDC, Pakistan) ou encore les principaux pays d'accueil des ressortissants de ces pays (le Kenya et l'Éthiopie pour les Somaliens, le Pakistan pour les Afghans, la Jordanie pour les Irakiens). Il est vraisemblable que la politique d'aide au développement soit influencée par lorigine des plus grands groupes de réfugiés arrivés récemment au Canada. En effet, tous les pays d'où sont originaires la majorité des nouveaux réfugiés au Canada font partie, à l'exception de la Chine, du Tchad et de la Somalie, des pays en développement ciblés par l'aide bilatérale du Canada ou identifiés comme partenaires du développement depuis 2014. La Somalie n'est pas directement ciblée mais le Kenya, pays d’accueil principal des réfugiés somaliens, l'est à titre de partenaire du développement.

Le financement croissant au HCR pourrait aussi s'inscrire dans des politiques visant à limiter les arrivées de réfugiés au Canada dans la mesure où l'action du HCR peut être décrite comme participant au confinement des populations, 
notamment en raison de l'ascendance des pays donateurs ${ }^{52}$. À cet effet, Whitaker ${ }^{3}$ souligne que les priorités du HCR ont été influencées par les priorités politiques et sécuritaires des États et que le HCR doit choisir entre son mandat de protection des réfugiés et des considérations politiques. Les donateurs, quant à eux, ont choisi de financer stratégiquement les programmes humanitaires dans les premiers pays d'asile, notamment pour éviter d'avoir à accueillir euxmêmes de grands nombres de réfugiés, ou de financer des interventions auprès de populations déplacées à l'intérieur de leur propre pays ou des opérations de rapatriement. La promotion de solutions régionales gagne ainsi du terrain, et les États et les organisations internationales témoignent d'un intérêt croissant pour l'assistance et la protection des déplacés internes, avec des standards moins élevés 54 , au point d'y voir un substitut, plutôt qu'un complément, à la protection des réfugiés 55 . Celle évolution a fait écrire à Hyndman que le régime international de protection des réfugiés était passé de la défense du droit d'asile à celui de rester chez soi ${ }^{56}$, alors que Scheel et Ratfisch ont décrit le HCR comme une "police globale des populations $" 57$.

Il semble cohérent de relier l'augmentation du financement canadien au HCR, mais également sa nature de plus en plus liée à certaines opérations, à la volonté exprimée par le pays de prévenir l'arrivée de populations réfugiées au Canada. Fournir de l'aide sur place aux personnes déplacées ou dans le premier pays d'asile, tout comme adopter des mesures de dissuasion fortes, pourrait ainsi avoir pour objectif de limiter l'accès de demandeurs d'asile au territoire canadien.

\section{Un vote de méfiance envers le $\mathrm{HCR}$ ?}

Le politologue Väyrynen ${ }^{58}$ rapporte une tendance, à partir du début des années 1990, à allouer des fonds à des programmes spécifiques plutôt qu'au HCR dans son ensemble, et soutient que cela reflète la volonté des gouvernements d'influencer l'agence en fonction de leurs propres intérêts politiques. Or, ajoute-t-il, cette volonté de contrôle ne traduit pas seulement le désir des États de mener leur politique étatique à travers le HCR, mais également leur absence de confiance envers l'agence et sa capacité à mettre en œuvre ses programmes, à définir les priorités et à y répondre adéquatement en utilisant au mieux les ressources qui lui sont allouées. Dans le cas qui nous intéresse, si le financement affecté traduit effectivement la méfiance de l'État envers le HCR ou encore une position critique envers l'agence, cette méfiance ne prévaut pas seulement envers cette agence, mais caractérise la relation canadienne à l'ensemble des Nations Unies. En effet, le financement canadien à l'oNu est, dans son ensemble, encore plus affecté que le financement au HCR (il passe de $75 \%$ en 2006 à $86 \%$ en 2013). À cet égard, Smille et Minear soulignent que plusieurs donateurs disent affecter leur financement à des projets ou des pays en particulier, et faire un suivi et une évaluation des projets de plus en plus rigoureuse en raison d'un manque de confiance envers les organisations humanitaires ${ }^{59}$. La méfiance ne peut, néanmoins, quêtre limitée: si l'affectation croissante du financement pourrait traduire une certaine méfiance, l'augmentation importante de la contribution canadienne au HCR au fil des ans semble plutôt être une marque de confiance envers l'organisation.

\section{Éléments de conclusion}

Lanalyse des éléments qui influencent le financement du Canada au HCR nous conduit à une conclusion mixte: la contribution canadienne au HCR n'est pas parfaitement désintéressée et relève non seulement de préoccupations humanitaires, mais aussi d'une politique intérieure et extérieure, ce qui est cohérent si l'on considère que ces axes politiques sont liés et que les priorités de la politique intérieure influencent fortement la politique extérieure.

Il existe une certaine coïncidence entre les pays visés par l'aide canadienne au HCR, les pays ciblés par les politiques canadiennes de développement et l'origine des plus grands groupes de réfugiés arrivés au Canada ces dernières années, ce qui rejoint les observations de Smillie et Minear, Loescher, Betts et Milner ou Bermeo et Leblang60. Or, le fait de cibler les opérations du HCR dans ces pays peut aussi s'expliquer par les besoins importants liés à l'assistance et la protection de réfugiés et de déplacés internes en ces lieux. Il $\mathrm{y}$ a, en effet, une convergence entre les besoins humanitaires et des considérations migratoires et d'asile. Il est dès lors complexe de déterminer avec exactitude les motivations qui sous-tendent la politique de financement au HCR, d'autant que ces motivations sont probablement plurielles, combinant des intérêts liés à la politique étrangère et intérieure, ainsi que la volonté de participer à l'effort humanitaire, donc de respecter les engagements internationaux canadiens, et possiblement une réticence à faire entièrement confiance aux Nations Unies, ce qui est une tendance observable chez plusieurs donateurs. Le facteur humain qui intervient nécessairement dans la prise de décisions doit, quant à lui, être mieux compris et cerné.

Il demeure que ce financement semble témoigner au moins en partie d'une volonté politique de contrôler les actions du HCR, puisque son affectation s'écarte des priorités établies par l'agence. Cette conclusion n'est guère surprenante. Elle corrobore ce qui a été observé à une échelle mondiale. L'influence du Canada sur le HCR par l'entremise de son financement est sans doute limitée, sa contribution annuelle représentant moins de $2 \%$ du budget de l'agence. La constance de ce financement témoigne cependant de l'engagement du pays et lui confère vraisemblablement une autorité morale. 
Labsence de conclusion définitive de cette recherche s'explique en partie par le fait qu'il s'agit de l'une des premières à s'intéresser aux logiques qui sous-tendent le financement canadien au HCR. D’autres études et des entretiens avec les décideurs seront nécessaires pour clarifier les motivations politiques et mieux interpréter les liens entre la politique d'aide internationale du Canada et sa politique d'immigration et d'aide aux réfugiés. Il serait intéressant de tenter de cerner ce qui, dans les choix de financement, pourrait se rattacher à l'idéologie du parti Conservateur et au pouvoir durant la période étudiée, et quels facteurs ont particulièrement influencé le financement affecté durant cette période, notamment en considérant les pistes proposées par Smillie et Minear ${ }^{61}$. En plus de tenir compte de considérations migratoires et commerciales, il conviendrait alors d'examiner les liens historiques, la proximité géographique, la politique régionale et les préoccupations sécuritaires du Canada. Enfin, une comparaison systématique avec l'évolution du financement des autres principaux pays donateurs serait indispensable pour identifier d'éventuelles spécificités dans la contribution canadienne.

\section{Notes}

1 À moins d'indications contraires, tous les montants exprimés sont en dollars canadiens et ont été ajustés en utilisant l'indice canadien des prix à la consommation et en prenant 2013 comme année de référence, voir http://www.banqueducanada.ca/taux/renseignements-complementaires/ feuille-de-calcul-de-linflation/.

2 Rapports statistique sur l'aide internationale de l'Agence canadienne de développement international pour les années financières 2006-2007 à 2012-2013; Rapport statistique sur l'aide internationale du Canada; Exercice 20132014 (Ministère des Affaires étrangères, du commerce et développement, 2015); "Réponse du ministre des Affaires étrangères, John Baird, à une demande de renseignement au gouvernement sur le financement et la participation $\mathrm{du}$ Canada au sein de l'organisation des Nations Unies formulée par la députée de Laurier/Sainte-Marie, Hélène Laverdière» (Gouvernement du Canada, 2014).

3 Nous utilisons la notion de financement affecté en référence aux budgets du gouvernement canadien qui sont affectés à des projets spécifiques du HCR, par opposition à des financements non affectés dont le HCR peut disposer librement, selon ses priorités.

4 David Morin et Stéphane Roussel, «Autopsie de la politique étrangère de Stephen Harper: un examen préliminaire ", Canadian Foreign Policy Journal 20, nº. 1 (2014): 1-8.

5 Steven D. Roper et Lilian A. Barria, «Burden Sharing in the Funding of the UNHCR: Refugee Protection as an Impure Public Good», Journal of Conflict Resolution 54, $\mathrm{n}^{\circ} .4$ (2010) : 616-37.
6 «Funding Dilemmas in Refugee Assistance: Political Interests and Institutional Reforms in UNHCR", International Migration Review 35, $\mathrm{n}^{\circ} .1$ (2001): 143-67.

7 Ibid.

8 UNHCR: The Politics and Practice of Refugee Protection into the Twenty First Century (New York: Routledge, 2008).

9 Judith Randel et Tony German, "Trends in the Financing of Humanitarian Action", in The New Humanitarianisms: A Review of Trends in Global Humanitarian Action, dir. Joanna Macrae (Londres: Overseas Development Institute, 2002), 19-28.

10 The Quality of Money; Donor Behavior in Humanitarian Financing (Somerville, MA: Humanitarianism and War Project, Tufts University, 2003).

11 Jennifer Hyndman, Managing Displacement: Refugees and the Politics of Humanitarianism (Minneapolis, MN; Londres: University of Minnesota Press, 2000); Liisa Malkki, «Refugees and Exile: From "Refugee Studies" to the National Order of Things", Annual Review Anthropo$\log y 24$ (1995a) : 495-523.

12 Christopher Lee, «Humanitarian Assistance as Containment; New Codes for a New Order", Working Paper Series, $n^{o} .72$ (Oxford: Refugee Studies Centre, University of Oxford, 2010); Abby Stoddard, International Humanitarian Financing: Review and Comparative Assessment of Instruments; a Study for the Good Humanitarian Donorship Initiative Commissioned by the Office of us Foreign Disaster Assistance (Londres: Humanitarian Outcomes, 2008).

13 Ather H., Akbari et Martha MacDonald, «Immigration Policy in Australia, Canada, New Zealand, and the United States: An Overview of Recent Trends ", International Migration Review 48, $\mathrm{n}^{\circ} .3$ (2014): 801-22; Amrita Hari, «Temporariness, Rights, and Citizenship: The Latest Chapter in Canada's Exclusionary Migration and Refugee History», Refuge 30, $\mathrm{n}^{\circ} .2$ (2014) : 35-44; Dagmar Soennecken, «Shifting up and Back; the European Turn in Canadian Refugee Policy", Comparative Migration Studies 2, $\mathrm{n}^{\circ} .1$ (2014): 101-22; Raimo Väyrynen, «Funding Dilmmas in Refugee Assistance: Political Interests and Institutional Reforms in UNHCR », International Migration Review 35, $\mathrm{n}^{\circ} .1$ (2001): 143-67.

14 "Aide humanitaire», Ministère des Affaires étrangères, commerce et développement Canada, http://www.international.gc.ca/development-developpement/humanitarian_ response-situations_crises/iha-ahi.aspx?lang=fra.

15 Gabriel Goyette, «Charity Begins at Home: The Extractive Sector as an Illustration of Changes and continuities in the New De Facto Canadian Aid Policy", in Rethinking Canadian Aid, dir. Stephen Brown, Molly den Heyer et David R. Black (Ottawa: University of Ottawa Press, 2014), 242-259.

16 Justin Massie et Stéphane Roussel, «Preventing, Substituting, or Complementing the Use of Force? Development Assistance in Canadian Strategic Culture», in Rethinking Canadian Aid, dir. Stephen Brown, Molly den Heyer et David R. Black (Ottawa: University of Ottawa Press, 2014), 143-16o. 
17 Francois Audet, Francis Paquette et Stéfanie Bergerone, «Religious nongovernmental organisations and Canadian international aid, 2001-2010: a preliminary study", Canadian Journal of Development Studies/Revue canadienne détudes du développement, 34, nº. 2 (2013) : 291-320.

18 Ryan Macdonald et John F. F. Hoddinott, «Determinants of Canadian bilateral aid allocations: humanitarian, commercial or political?" Canadian Journal of Economics/ Revue canadienne d'économique, 37, $\mathrm{n}^{\circ} .2$ (2004): 294-312; François Audet et Olga Navarro-Flores, «The Management of Canadian Development Assistance: Ideology, Electoral Politics or Public Interest? ", in Rethinking Canadian Aid, dir. Stephen Brown, Molly den Heyer et David R. Black (Ottawa: University of Ottawa Press, 2014), 179194; Gabriel C. Goyette et Francois Audet, « La politique publique d'aide humanitaire canadienne: entre croissance et incohérence", Canadian Journal of Development Studies / Revue canadienne d'études du développement, 37, $\mathrm{n}^{\mathrm{o}} .3$ (2016) (3) (version en ligne).

19 Tom J. Farer, Daniele Archibugi, Chris Brown, Neta C. Crawford, Thomas G. Weiss et Nicholas J. Wheeler, «Roundtable: Humanitarian Intervention After 9/11", International Relations 19, $\mathrm{n}^{\circ} .2$ (2005): 211-250; Francois Audet, «Lacteur humanitaire en crise existentielle: les défis du nouvel espace humanitaire», Études Internationales, 42, $\mathrm{n}^{\mathrm{o}} .4(2011): 447-472$.

20 Éric Marclay, «Le virage vers les questions de sécurité de l'aide publique au développement», in Laide canadienne au développement, dir. François Audet, Marie-Eve Desrosiers, Stéphane Roussel (Montréal: Les Presses de l'Université de Montréal, 2008), 241-263.

21 Question Q-598, 9 juin 2014.

22 Ces documents sont intitulés «Rapport statistique sur l'aide internationale». Les rapports produits pour les années financières 2006-2007, 2007-2008, 2008-2009, 2009-2010, 2010-2011, 2011-2012 et 2012-2013 ont été consultés.

23 "Rapport statistique sur lraide internationale du Canada; Exercice 2013-2014" (Gatineau: Ministère des Affaires étrangères, du Commerce et du Développement, 2015).

24 http://www.international.gc.ca/development-developpement/aidtransparency-transparenceaide/browser-banque .aspx?lang=fra.

25 Les «Profils des donateurs» des éditions 2006 à 2013 du Rapport Global du HCR ont été consultés.

26 Canada-Admissions de résidents permanents tels que réfugiés selon les dix principaux pays de la citoyenneté, de 2006 à 2014 (Citoyenneté et immigration Canada, 29 juillet 2015).

27 La base de données du Financial Tracking Service inclut toutes les contributions humanitaires qui lui sont rapportées par les donateurs ou les organisations bénéficiaires, dont les agences des Nations Unies, les organisations non gouvernementales ou les Sociétés nationales de la CroixRouge et du Croissant Rouge.
28 "Funding UNHCR's Programmes", in UNHCR Global Report 2013 (Genève: Haut Commissariat des Nations Unies pour les réfugiés, 2014), 106-30.

29 "Profils des donateurs", dans les éditions 2006 à 2013 du Rapport Global du HCR (Genève: Haut Commissariat des Nations Unies pour les réfugiés).

30 «Funding UNHCR's Programmes", in UNHCR Global Report 2013 (Genève: Haut Commissariat des Nations Unies pour les réfugiés, 2014), 106-30.

31 «Identifying Needs and Funding Requirements», in UNHCR Global Appeal 2010-11 (Genève: Haut Commissariat des Nations Unies pour les réfugiés, sans date), 66-75.

32 La corrélation ne peut être établie de façon certaine puisque les fiches de projets du MAECD ne précisent pas si le financement a été accordé en raison d'un appel d'urgence ou de l'appel annuel. Mises en relation avec les appels spéciaux, les dates auxquelles le financement est accordé permettent toutefois de déduire que certains financements ont été octroyés en vertu d'un appel donné. Cette information est par ailleurs parfois disponible sur le site du Financial Tracking Service du Bureau de coordination des affaires humanitaires des Nations Unies (https://fts.unocha.org).

33 Rapport du vérificateur général du Canada: Répondre aux crises humanitaires internationales émergentes (Bureau du Vérificateur général du Canada, 2014). http://www.oag-bvg .gc.ca/internet/docs/parl_oag_201411_o1_f.pdf.

34 Par exemple, dans The Charity of Nations; Humanitarian Action in a Calculating World, Smillie et Minear soulignent qu'un fonctionnaire de l'ACDI a largement influencé la politique d'aide alimentaire du Canada en réduisant l'aide alimentaire en faveur de compléments, comme des vitamines (Bloomfield: Kumarian Press, 2004).

35 Patrick Hassenteufel, Sociologie politique: l'action publique (Paris: Armand Colin, 2011).

36 Selon Smillie et Minear (The Charity of Nations), les médias jouent un rôle éventuel lorsque les intérêts stratégiques ne sont pas un déterminant majeur.

37 Ian Smille et Larry Minear, The Charity of Nations; Raimo Väyrynen, "Funding Dilmmas»; Gil Loescher, Alexander Betts et James Milner, UNHCR: The Politics and Practice of Refugee Protection into the Twenty First Century (New York: Routledge, 2008).

38 Barry Bozeman, Public Values and Public Interest (Washington: Georgetown University Press, 2007).

39 «Plan d'action sur l'efficacité de l'aide: au cœur de l'aide internationale du Canada.» Ministère des Affaires étrangères, commerce et développement Canada, http://www international.gc.ca/development-developpement/priorities-priorites/aidagenda-planaide.aspx?lang=fra.

40 «Le Canada met à jour sa liste de pays en développement ciblés.» Ministère des Affaires étrangères, commerce et développement Canada, 2014. http://www.international .gc.ca/media/dev/news-communiques/2014/o6/27abg .aspx?lang=fra (page archivée dans le Web). 
41 «ésultats stratégiques et Architecture des activités de programme (en vigueur au 1er avril 2011) », Citoyenneté et immigration Canada, http://www.cic.gc.ca/francais/ministere/ aap/2011/activite-43.asp (page archivée dans le Web).

42 Gil Loescher, Alexander Betts et James Milner, UNHCR: The Politics and Practice of Refugee Protection.

43 «Profil de projet: Soutenir la Stratégie globale de solutions du HCR pour vaincre les situations de réfugiés prolongées ", Banque de projets de développement international, Affaires étrangères, commerceetdéveloppementCanada,http://www .acdi-cida.gc.ca/cidaweb/cpo.nsf/vWebProjByPartnerFr/ D467BD3813CA570F85257C80003B295D.

44 Ather H., Akbari et Martha MacDonald, «Immigration Policy in Australia, Canada, New Zealand, and the United States»; Jennifer Hyndman et Alison Mountz, "Another Brick in the Wall? Neo-Refoulement and the Externalization of Asylum by Australia and Europe », Government and Opposition 43, $\mathrm{n}^{\mathrm{o}} .2$ (2008): 249-69; Dagmar Soennecken, "Shifting up and Back; the European Turn in Canadian Refugee Policy».

45 «Résultats stratégiques et Architecture des activités de programme (en vigueur au 1er avril 2011) », Citoyenneté et immigration Canada, http://www.cic.gc.ca/francais/ministere aap/2011/activite-43.asp (page archivée dans le Web).

46 Andrew Brouwer et Judith Kumin, «Interception and Asylum: When Migration Control and Human Rights Collide, » Refuge 21, nº. 4 (2003) : 6-24; Janet Dench et François Crépeau, "Interdiction at the Expense of Human Rights: A Long-Term Containment Strategy", Refuge 21, $\mathrm{n}^{\mathrm{O}} \cdot 4$ (2003) : 2-5.

47 Y.Z. c. Canada (Citoyenneté et Immigration), décision de la Cour fédérale, 2015 CF 892.

48 New Refugee System - One Year On (Montréal: Conseil canadien pour les réfugiés, 2013).

49 Par exemple, Gil Loescher, Alexander Betts et James Milner, UNHCR: The Politics and Practice of Refugee Protection; Ian Smille et Larry Minear, The Charity of Nations.

50 «Migration and Foreign Aid», International Organization 69 (2015): 627-57.

51 «Canada - Admissions de résidents permanents tels que réfugiés selon les dix principaux pays de la citoyenneté, de
2006 à 2014 ", Citoyenneté et immigration Canada, 29 juillet 2015 (données compilées à notre demande).

52 Christopher Lee, «Humanitarian Assistance as Containment».

53 Beth Elise Whitaker, «Funding the International Refugee Regime: Implications for Protection», Global Governance 14 (2008) : 241-58.

54 Jean-Hervé Bradol, "Caring for Health» in Humanitarian Negotiations Revealed; the MSF Experience, dir. Claire Magone, Michaël Neuman et Fabrice Weissman (Paris: Médecins Sans Frontières, 2011), 227-50.

55 Cécile Dubernet, "Quand l'espace humanitaire devient une zone de guerre: personnes déplacées et peurs sécuritaires", Asylon(s), revue électronique du réseau Terra $\mathrm{n}^{\circ} .4$ (2006). Jennifer Hyndman, Managing Displacement. Katy Long, «No Entry! A Review of UNHCR's Response to Border Closures in Situations of Mass Refugee Influx », in New Issues in Refugee Research (Genève: Policy Development and Evaluation Service, UNHCR, 2010).

56 Managing Displacement.

57 Stephan Scheel et Philipp Ratfisch, «Refugee Protection Meets Migration Management: UNHCR as a Global Police of Populations ", Journal of Ethnic and Migration Studies 40, $\mathrm{n}^{\mathrm{o}} .6$ (2014): 924-41.

58 Raimo Väyrynen, «Funding Dilmmas in Refugee Assistance».

59 "The Quality of Money.»

6o Ian Smille et Larry Minear, The Charity of Nations; Gil Loescher, Alexander Betts et James Milner, UNHCR: The Politics and Practice of Refugee Protection; Sarah Blodgett Bermeo et David Leblang. "Migration and Foreign Aid.» International Organization 69 (2015): 627-57;

61 The Charity of Nations.

Catherine-Lune Grayson, chercheuse post-doctorale, McGill. Lauteur peut être contactée à lunegrayson@gmail.com.

François Audet, professeur agrégé, École des sciences de la gestion, Université du Québec à Montréal. L'auteur peut être contactéàaudet.francois@uqam.ca.

(C) Catherine-Lune Grayson et François Audet, 2017. Cette œuvre en libre accès fait l'objet d'une licence Creative Commons Attribution-NonCommercial 4.0 International License, laquelle autorise l'utilisation, la reproduction et la distribution de l'œuvre sur tout support à des fins non commerciales, pourvu que l'auteur ou les auteurs originaux soient mentionnés et que la publication originale dans Refuge : revue canadienne sur les réfugiés soit citée. 